\title{
The 10th International Congress on Cutaneous Adverse Drug Reactions, Shimane, Japan, 2018: Focus on New Discoveries
}

\author{
Cristina Olteanu ${ }^{1} \cdot$ Neil H. Shear ${ }^{2,3} \cdot$ Eishin Morita ${ }^{4} \cdot$ Wen-Hung Chung ${ }^{5,6} \cdot$ Hiroyuki Niihara $^{4} \cdot$ Setsuko Matsukura ${ }^{7,8}$. \\ Rena Hashimoto ${ }^{9} \cdot$ Roni P. Dodiuk-Gad ${ }^{2,10,11}$
}

Published online: 29 April 2019

(c) Springer Nature Switzerland AG 2019

\section{Introduction}

The first International Congress on Cutaneous Adverse Drug Reactions took place in Paris, France, in 1994. Latterly, the 10th International Congress on Cutaneous Adverse Drug Reactions was held at the Shimane Civic Center in Shimane, Japan, on 10-11 November 2018, as part of the 70th Annual Meeting of the Western Division of Japanese Dermatological Association. This meeting was of special significance because it was the first time the congress had taken place in Japan.

The meeting was organized by the co-chairs of the congress: Professor Eishin Morita, Chief Professor of Dermatology, Shimane University Faculty of Medicine, Izumo, Japan, and also president of the 70th Annual Meeting of the Western Division of Japanese Dermatological Association; and Professor Wen-Hung Chung, Director of Drug Hypersensitivity Clinical and Research Center, Chang Gung Memorial

Electronic supplementary material The online version of this article (https://doi.org/10.1007/s40264-019-00818-1) contains supplementary material, which is available to authorized users.

Roni P. Dodiuk-Gad

rdodiukgad@gmail.com

1 Division of Dermatology, Department of Medicine, University of Alberta, Edmonton, AB, Canada

2 Division of Dermatology, Department of Medicine, Sunnybrook Health Sciences Centre, University of Toronto, Toronto, ON, Canada

3 Division of Clinical Pharmacology and Toxicology, Department of Medicine, University of Toronto, Toronto, ON, Canada

4 Department of Dermatology, Shimane University Faculty of Medicine, Izumo, Japan

5 Department of Dermatology, Drug Hypersensitivity Clinical and Research Center, Chang Gung Memorial Hospitals, Linkou, Taipei, Taiwan
Hospital, and Professor and Head of Dermatology at Chung Gang University College of Medicine, Taoyuan, Taiwan.

During the meeting, a book launch on the first book on the topic of cutaneous adverse drug reactions (cADRs) Advances in Diagnosis and Management of Cutaneous Adverse Drug Reactions: Current and Future Trends, by Springer-Nature - was conducted. The editors of this book are Dr. Neil H. Shear and Dr. Roni P. Dodiuk-Gad, and the editorial assistants are Dr. Cristina Olteanu and Dr. Rena Hashimoto. This book can be found at the following webpage: https://www.springer.com/la/book/9789811314889.

Participants of the scientific meeting attended from the following countries: Germany, Spain, Switzerland, The Netherlands, USA, Canada, Israel, Taiwan, Malaysia, China, Mongolia, Singapore, Thailand, and Japan. During the meeting, international leaders on the topic of cADR presented their breakthrough research and much discussion was conducted on the translation of scientific knowledge into practical, clinical guidelines, with a focus on new discoveries and future directions. We would like to present the most important studies presented during the meeting and also the newest questions that have arisen. The sessions of iSCAR

6 College of Medicine, Chang Gung University, Taoyüan, Taiwan

7 Department of Dermatology, Saiseikai Yokohamashi Nanbu Hospital, Yokohama, Japan

8 Department of Environmental Immuno-Dermatology, Yokohama City University Graduate School of Medicine, Yokohama, Japan

9 Division of Dermatology, Keio University Hospital, Tokyo, Japan

10 The Ruth and Bruce Rappaport Faculty of Medicine, Technion Institute of Technology, Haifa, Israel

11 Department of Dermatology, Emek Medical Centre, Afula, Israel 
2018 were not created according to topics, and each session included talks on different fields, with no common theme. However, for the convenience of the reader, this summary of the meeting was designed according to various topics on cADRs gathered from different sessions.

\section{Pharmacogenomics}

Professor Elizabeth Phillips, Vanderbilt University Medical Center, Nashville, TN, USA, discussed that the therapeutic recommendations for HLA-B*15:02 screening are now also applicable to oxcarbazepine; it is recommended to not use oxcarbazepine if the patient is HLA-B*15:02 positive and oxcarbazepine-naïve. If the HLA-B*15:02 genotype is negative, then patients can use oxcarbazepine as per the regular doses recommended. Other aromatic anticonvulsants, including phenytoin, phenobarbital, lamotrigine, eslicarbazepine, and fosphenytoin, have limited indications that link Stevens-Johnson syndrome/toxic epidermal necrolysis (SJS/TEN) with the HLA-B*15:02 allele. Nevertheless, it is advisable to be cautious when selecting a different aromatic anticonvulsant [1].

Professor Elizabeth Phillips also presented a translational laboratory-to-bedside research success story-the discovery of HLA-B*57:01-associated abacavir hypersensitivity. Using a mouse model, it was discovered that $\mathrm{CD} 4+\mathrm{T}$ cells have a major role in induction of tolerance to a modified endogenous peptide repertoire of the HLA-B*57:01 allele when combined with abacavir. Hence, some who carry the HLA-B*57:01 allele do not have adverse drug reactions to abacavir. This discovery also allows for future studies in the field of T-cell-mediated drug tolerance [2].

Professor Neil H. Shear, University of Toronto, Canada, emphasized that knowledge and awareness of at-risk ethnicities, genetic associations and screening, as well as usage guidelines, have been found to be limited in general physicians and physicians of other specialties, other than dermatology, in a Canadian academic institution; however, interest in learning about these topics is high.

Professor Bruce C. Carleton, Pediatrics, Division of Translational Therapeutics, Faculty of Medicine, University of British Columbia, Pharmaceutical Outcomes Programme, British Columbia Children's Hospital, and Child Family Research Institute, Canada, presented the Canadian Pharmacogenomics Network for Drug Safety, which is a group of healthcare centers in Canada that were the first to confirm human leukocyte antigen (HLA) involvement in skin reactions in children due to carbamazepine.

\section{Stevens-Johnson Syndrome and Toxic Epidermal Necrolysis}

Professor Teresa Bellón, Hospital La Paz Health Research Institute, Spain, reported that biomarkers may aid for early detection of SJS/TEN prior to receiving the results of a skin biopsy. Granulysin is a key mediator responsible for disseminated keratinocyte death in patients with earlystage SJS/TEN compared with those with other druginduced skin reactions. Hence, the serum granulysin test can provide a rapid diagnosis of SJS/TEN [3, 4].

Professor Wen-Hung Chung, Department of Dermatology, Drug Hypersensitivity Clinical and Research Center, Chang Gung Memorial Hospital, Taipei, Linkou and Keelung, Taiwan, explained that in exploring cytokines and chemokines with prognostic potential in SJS/TEN, higher levels of granulysin and the cytokine interleukin (IL)-15 are associated with more severe disease. In patients with SJS/TEN, IL-15 was also found to have an association with mortality. Furthermore, IL-15 induces cytotoxic T lymphocyte responses as well as natural killer (NK) cells. The discoveries of the various roles of IL- 15 points to its possible application in monitoring prognosis, as well as interventions in SJS/TEN [5].

Professor Haur Yueh Lee, Department of Dermatology, Singapore General Hospital, and Allergy Centre, Singapore General Hospital, Singapore, reported that strontium ranelate, an anti-osteoporotic medication registered in the European Union in 2004, was found to have increased risk of both drug reaction with eosinophilia and systemic symptoms (DRESS) and SJS/TEN. HLA analysis suggests that two alleles, HLA-B*58:01 and HLA-A*33:03, are genetic risk factors for inducing SJS/TEN in Asian patients consuming strontium ranelate [6].

Professor Tsu-Man Chiu, Division of Dermatology, Department Changhua Christian Hospital, Taiwan, described an analysis from multiple Asian countries that found new potential drug causes of SJS/TEN in Asian populations: strontium ranelate, cyclooxygenase (COX)-2 inhibitors, oxcarbazepine, and sulfasalazine. In addition, quinolones were also found to be a common cause. Several drugs (sorafenib, oseltamivir, isotretinoin, and terbinafine) labeled as carrying a risk for SJS/TEN by the US FDA were not found to be a risk factor for inducing SJS/TEN in the Asian countries investigated in this research [7].

Professor Chun-Wei Lu, Department of Dermatology, Drug Hypersensitivity Clinical and Research Center, Chang Gung Memorial Hospital, Linkou, Taiwan, explained that in terms of treatments, the anti-tumor necrosis factor (TNF)-á biologic agent etanercept has been found to serve as an effective alternative to corticosteroids in SJS/TEN in terms of reducing the skin healing time and 
decreasing the TNFá and granulysin secretions in blister fluid and plasma, as well as increasing the regulatory $\mathrm{T}$ cell (Treg) population [4].

Professor Roni P. Dodiuk-Gad, Technion Institute of Technology, Israel, and the University of Toronto, Canada, reported that survivors of SJS/TEN have severe long-term psychological complications, decreased health-related quality of life [8], and physical complications, and require ongoing medical follow-up [9].

Professor Chun-Bing Chen, Department of Dermatology, Drug Hypersensitivity Clinical and Research Center, Chang Gung Memorial Hospital, Keelung, Taipei, Linkou, and Chang Gung University College of Medicine, Taoyuan, Taiwan, explained that lupus erythematosus mimicking TEN is a specific cutaneous manifestation of lupus erythematosus. A longer clinical course, positive antinuclear antibodies (ANAs), decreased $\mathrm{C} 3$ or $\mathrm{C} 4$, positive lupus band test, and periadnexal infiltration or melanin incontinence in histopathology help to differentiate lupus erythematosus from TEN.

Professor Miho Mukai, Keio University Department of Dermatology, presented a case of a person who developed psoriasis on scars following TEN and suggested that re-epithelialization and ethanol injection might contribute to the characteristic course of psoriatic lesions.

Professor Mayumi Ueta, Department of Frontier Medical Science and Technology for Ophthalmology, Kyoto Prefectural University of Medicine, Kyoto, Japan, explained that medications including cold medicines and nonsteroidal antiinflammatory drugs (NSAIDS) were the most common causative drugs for SJS/TEN with severe ocular complications.

\section{Drug-Induced Hypersensitivity Syndrome/ Drug Reaction with Eosinophilia and Systemic Symptoms}

Professor Tatsuya Katsumi, Department of Dermatology, Niigata University Graduate School of Medical and Dental Sciences, Niigata, Japan, described a new severity score for drug-induced hypersensitivity syndrome (DiHS)/drug reaction with eosinophilia and systemic symptoms (DRESS) as a useful tool. This scoring system also made it possible for predicting complications induced by cytomegalovirus (CMV) and when to consider early treatment with anti-CMV drugs [10]. He also emphasized that in patients with DRESS, a small skin ulcer could be an initial sign of CMV infection before the CMV antigen became positive.

Professor Anna Nakajima, Nara Medical University, Japan, reported a case of drug eruption resembling DRESS that developed 5 months after beginning lamotrigine. The author suggested that the lag period due to lamotrigineinduced DRESS can be very long.
Professor Siew Eng Choon, Hospital Sultanah Aminah Johor Bahru, Johor, Malaysia, reported that commonly implicated medications in severe cutaneous drug reactions include allopurinol, anticonvulsants (phenytoin and carbamazepine), antibiotics, and NSAIDs.

Professor Hideo Asada, Department of Dermatology, Nara Medical University of Medicine, Japan, explained that thymus and activation-regulated chemokine (TARC; a $\mathrm{T}$ helper [Th] 2-associated chemokine) levels in serum are higher in the acute stages of DRESS than in other drug eruptions, providing a marker for early diagnosis of DRESS.

\section{Molecular Mechanisms in Adverse Drug Reactions}

Professor Riichiro Abe, Niigata University Graduate School of Medical and Dental Sciences, Japan, presented a new term, 'necroptosis', to describe necrotic cell death that can be driven by molecular pathways and is dependent on receptor-interacting protein kinase-3 (RIPK3) [11]. Necroptosis is derived from the two known terms describing two different forms of cell death-necrosis and apoptosis. Necrosis is a mechanism of cell death that is a cause or consequence of disease, while apoptosis is driven by a set of molecular mechanisms that 'program' cells to die. Necroptosis is suggested to be induced by interactions of formyl peptide receptor 1 (FPR1) and annexin A1, and may contribute to SJS/ TEN development. Hence, the necroptosis pathway can be a drug target for treating patients with SJS/TEN [12].

Professor Shuen-Iu Hung, Department and Institute of Pharmacology, School of Medicine, National Yang-Ming University, Taipei, Taiwan, discussed that T-cell receptor (TCR) clonotypes are implicated in the pathophysiology of several drug reactions also involving HLA alleles. It has been shown that there is a need for a specific T-cell repertoire, in addition to a specific HLA allele, to develop a severe drug reaction. Hence, this could explain why not all HLA-B*15:02 carriers have a reaction to carbamazepine. The drug-specific TCRs of cytotoxic T-cell lymphocytes have key roles in inducing SJS/TEN [13]. In addition, it has been found that Th17 cells could be involved in inducing SJS/TEN. These major findings provide us with insight into the pathogenesis of, and treatment possibilities for, SJS/TEN [14].

Professor Teresa Bellón, Hospital La Paz Health Research Institute, Spain, reported that in DiHS/DRESS, there is a slow transition from Treg to Th17 cell expansion that is mediated by different subtypes of monocytes (MOs) that vary during the early and prolonged latent period of DRESS. In the early stages of DRESS, MOs are more likely to expand induced Tregs (iTregs) due to the increase in CD14+classical MOs. However, at the later stages, there is induction of Th17 cell 
development due to CD16 patrolling MOs that produce the cytokine IL-6. Hence, one potential therapeutic target for DiHS/DRESS is the particular subtype of MOs that can influence either Treg or Th17 cell expansion [15].

Professor Sylvia H. Kardaun, Department of Dermatology, Isala-Diaconessenhuis, Meppel, The Netherlands, emphasized that it is very important to use validation scoring systems for various severe cutaneous adverse drug reaction (SCAR) subtypes, and suggested that application of these scoring systems indicate that true overlap is rare.

\section{Cancer Treatments and Toxicity}

Professor Chia-Yu Chu, Department of Dermatology, College of Medicine, National Taiwan University, Taiwan, explained that increasing attention is being given to anticancer therapies and the link between treatment outcome and cutaneous toxicity. In all three main classes of anticancer agents, skin toxicity is a potential biomarker for positive treatment response. The following are examples of such adverse effects: (1) molecularly targeted kinase inhibitors-hand/foot syndrome; (2) immune checkpoint inhibitors-pigmentary changes such as vitiligo; and (3) cytotoxic chemotherapeutics - alopecia. An increased awareness of these toxicities is important as it could provide recognition of the effectiveness of anticancer therapy and subsequently influence patient outcomes and further treatment decisions [16].

Professor Yi-Shuan Sheen, Department of Dermatology, National Taiwan University Hospital, and College of Medicine, National Taiwan University, Taiwan, discussed that epidermal growth factor receptor tyrosine kinase inhibitors (EGFR-TKIs) are associated with vascular adverse events. Use of these drugs can contribute to impairment of blood vessel walls through multiple factors, leading to vascular injury and inflammation [17].

Professor Lukas Flatz, University of Zurich, Switzerland, explained that new research findings suggest that uveitis may be a positive surrogate marker for a melanoma response to therapy in patients treated with pembrolizumab; uveitis is a very rare adverse effect occurring in approximately $1 \%$ of patients who are being treated with pembrolizumab. Accordingly, patients should have ophthalmological examinations, including optical coherence tomography, prior to the start of immunotherapy, and regular follow-up during treatment [18].

\section{Investigative Tests}

Professor Jettanong Klaewsongkram, Chulalongkorn University, Thailand, explained that drug causality assessment in cADR remains a challenge. Some testing options available include an in vivo drug patch test (DPT) and ex vivo interferon (IFN)-ã enzyme-linked immunospot (ELISpot) assay with lymphocyte transformation test (LTT). The culprit drug may be found by using both DPT and ELISpot tests [19].

Professor Pawinee Rerknimitr, Division of Dermatology, Department of Medicine, Faculty of Medicine, Skin and Allergy Research Unit, Chulalongkorn University, Bangkok, Thailand, presented findings that the T-cell activation assay is an alternative test for the LTT to screen for culprit drugs. The test sensitivity is around $80 \%$ and its specificity is around $95 \%$. The test comprises three different protocols to assess the expression of the various cytokines: flow cytometry assessing granulysin expression in CD4 + T cells; ELISpot assay measuring granzyme B production; and a cytokine bead array measuring IFN $\gamma$ levels in cell supernatant. Using the T-cell activation assay and the ALDEN score can increase the chances of identifying the culprit drug [20].

Professor Takayoshi Komatsu-Fujii, Department of Dermatology, Kyoto University School of Medicine, Kyoto, Japan, emphasized that activation-regulated chemokine (sTARC) levels and serum thymus are positively correlated with inflammation, and suggest prognostic value of sTARC for drug eruptions [21].

Professor Hiroyuki Niihara, Department of Dermatology, Shimane University Faculty of Medicine, Shimane, Japan, explained that an effective and useful tool is a new HLA-detecting method called loop-mediated isothermal amplification (LAMP) [22]. This has been tested for HLAB*58:01:01 and HLA-A*31:01:02 in the Japanese population, with both high sensitivity and specificity.

Professor Wichittra Tassaneeyakul, Department of Pharmacology, Faculty of Medicine, Khon Kaen University, Thailand, reported that a recent success of translational medicine in Thailand involves the HLA-B*15:02 test, which has been conducted for free for Thailand citizens since 1 October 2018, to prevent carbamazepineinduced SJS/TEN.

\section{Other Insightful Discussions}

Professor Maja Mockenhaupt, Medical Center and Medical Faculty, University of Freiburg, Germany, presented various epidemiological studies on SCAR, focusing on the studies investigating the frequency and risk factors of SCARs.

Professor John R. Stanley, Dermatology, University of Pennsylvania, Philadelphia, PA, USA, led an inspiring discussion, pointing out that many discoveries required only paying attention to simple observations, and also described some discoveries made throughout his career. 


\section{Conclusion}

iSCAR 2018 was a very important international scientific conference in which new discoveries and future directions were presented. Information on the scientific conference can be found on the official website, http://wjda70.jp/iscar.html (see electronic supplementary material for the iSCAR 2018 program).

Acknowledgements The authors thank Dr. Moran E. Barcan, MD, for assistance in the preparation of this manuscript.

\section{Compliance with Ethical Standards}

Funding No funding sources were used in the preparation of this manuscript.

Conflict of interest Cristina Olteanu, Neil H. Shear, Eishin Morita, Wen-Hung Chung, Hiroyuki Niihara, Setsuko Matsukura, Rena Hashimoto and Roni P. Dodiuk-Gad have no conflicts of interest to declare.

\section{References}

1. Phillips EJ, Sukasem C, Whirl-Carrillo M, et al. Clinical pharmacogenetics implementation consortium guideline for HLA genotype and use of carbamazepine and oxcarbazepine: 2017 update. Clin Pharmacol Ther. 2018;103:574-81.

2. Phillips EJ, Mallal SA. Active suppression rather than ignorance: tolerance to abacavir-induced HLA-B $* 57: 01$ peptide repertoire alteration. J Clin Investig. 2018;128:2746-9.

3. Lin YC, Sheu JN, Chung WH, et al. Vancomycin-Induced StevensJohnson Syndrome in a Boy Under 2 Years Old: An Early Diagnosis by Granulysin Rapid Test. Frontiers in pediatrics. 2018;6:26.

4. Wang CW, Yang LY, Chen CB, et al. Randomized, controlled trial of TNF-alpha antagonist in CTL-mediated severe cutaneous adverse reactions. J Clinl Investig. 2018;128:985-96.

5. Su SC, Mockenhaupt M, Wolkenstein P, et al. Interleukin-15 is associated with severity and mortality in stevens-johnson syndrome/toxic epidermal necrolysis. J Investig Dermatol. 2017;137:1065-73.

6. Lee HY, Lie D, Lim KS, et al. Strontium ranelate-induced toxic epidermal necrolysis in a patient with post-menopausal osteoporosis. Osteoporos Int. 2009;20:161-2.

7. Wang YH, Chen CB, Tassaneeyakul W, et al. The medication risk of stevens-johnson syndrome and toxic epidermal necrolysis in Asians: the major drug causality and comparison with the US FDA label. Clin Pharmacol Ther. 2019;105:112-20.

8. Dodiuk-Gad RP, Olteanu C, Feinstein A, et al. Major psychological complications and decreased health-related quality of life among survivors of Stevens-Johnson syndrome and toxic epidermal necrolysis. Br J Dermatol. 2016;175:422-4.

9. Olteanu C, Shear NH, Chew HF, et al. Severe physical complications among survivors of Stevens-Johnson syndrome and toxic epidermal necrolysis. Drug Saf. 2018;41:277-84.

10. Mizukawa Y, Hirahara K, Kano Y, et al. Drug-induced hypersensitivity syndrome (DiHS)/drug reaction with eosinophilia and systemic symptoms (DRESS) severity score: a useful tool for assessing disease severity and predicting fatal cytomegalovirus disease. J Am Acad Dermatol. 2019;80(3):670-8.

11. Linkermann A, Green DR. Necroptosis. New Engl J Med. 2014;370:455-65.

12. Saito N, Qiao H, Yanagi T, et al. An annexin A1-FPR1 interaction contributes to necroptosis of keratinocytes in severe cutaneous adverse drug reactions. Sci Transl Med. 2014;6:24595.

13. Ko TM, Chung WH, Wei CY, et al. Shared and restricted T-cell receptor use is crucial for carbamazepine-induced Stevens-Johnson syndrome. J Allergy Clin Immunol. 2011;128(1266-76):e11.

14. Teraki Y, Kawabe M, Izaki S. Possible role of TH17 cells in the pathogenesis of Stevens-Johnson syndrome and toxic epidermal necrolysis. J Allergy Clin Immunol. 2013;131:907-9.

15. Ushigome $\mathrm{Y}$, Mizukawa $\mathrm{Y}$, Kimishima M, et al. Monocytes are involved in the balance between regulatory T cells and Th17 cells in severe drug eruptions. Clin Exp Allergy. 2018;48:1453-63.

16. Rzepecki AK, Cheng H, McLellan BN. Cutaneous toxicity as a predictive biomarker for clinical outcome in patients receiving anticancer therapy. J Am Acad Dermatol. 2018;79:545-55.

17. Lacouture ME. Mechanisms of cutaneous toxicities to EGFR inhibitors. Nat Rev Cancer. 2006;6:803-12.

18. Diem S, Keller F, Ruesch R, et al. Pembrolizumab-triggered uveitis: an additional surrogate marker for responders in melanoma immunotherapy? J Immunother. 2016;39:379-82.

19. Klaewsongkram J, Sukasem C, Thantiworasit P, et al. Analysis of HLA-B allelic variation and IFN-gamma ELISpot responses in patients with severe cutaneous adverse reactions associated with drugs. J Allergy Clin Immunol Pract. 2019;7(219-27):e4.

20. Lin YC, Sheu JN, Chung WH, et al. Vancomycin-induced StevensJohnson syndrome in a boy under 2 years old: an early diagnosis by granulysin rapid test. Front Pediatr. 2018;6:26.

21. Komatsu-Fujii T, Chinuki Y, Niihara H, et al. The thymus and activation-regulated chemokine (TARC) level in serum at an early stage of a drug eruption is a prognostic biomarker of severity of systemic inflammation. Allergol Int. 2018;67:90-5.

22. Cheng SH, Kwan P, $\mathrm{Ng} \mathrm{HK}$, et al. New testing approach in HLA genotyping helps overcome barriers in effective clinical practice. Clin Chem. 2009;55:1568-72. 